\title{
Risk factors associated with cluster size of Mycobacterium tuberculosis (Mtb) of different RFLP lineages in Brazil
}

Renata Lyrio Peres ${ }^{1,2}$, Solange Alves Vinhas ${ }^{1}$, Fabíola Karla Correa Ribeiro ${ }^{1}$, Moisés Palaci ${ }^{1}$,

Thiago Nascimento do Prado², Bárbara Reis-Santos ${ }^{2,3}$, Eliana Zandonade², Philip Noel Suffys ${ }^{4}$, Jonathan E. Golub ${ }^{5}$, Lee W. Riley ${ }^{6}$ and Ethel Leonor Maciel ${ }^{2^{*}}$

\begin{abstract}
Background: Tuberculosis (TB) transmission is influenced by patient-related risk, environment and bacteriological factors. We determined the risk factors associated with cluster size of IS6110 RFLP based genotypes of Mycobacterium tuberculosis (Mtb) isolates from Vitoria, Espirito Santo, Brazil.

Methods: Cross-sectional study of new TB cases identified in the metropolitan area of Vitoria, Brazil between 2000 and 2010. Mtb isolates were genotyped by the IS6110 RFLP, spoligotyping and RD Rio . The isolates were classified according to genotype cluster sizes by three genotyping methods and associated patient epidemiologic characteristics. Regression Model was performed to identify factors associated with cluster size.

Results: Among 959 Mtb isolates, 461 (48\%) cases had an isolate that belonged to an RFLP cluster, and six clusters with ten or more isolates were identified. Of the isolates spoligotyped, 448 (52\%) were classified as LAM and 412 (48\%) as non-LAM. Our regression model found that 6-9 isolates/RFLP cluster were more likely belong to the LAM family, having the $\mathrm{RD}^{\text {Rio }}$ genotype and to be smear-positive (adjusted $\mathrm{OR}=1.17,95 \% \mathrm{Cl} 1.08-1.26$; adjusted $\mathrm{OR}=1$. 25, 95\% Cl 1.14-1.37; crude OR=2.68, 95\% IC 1.13-6.34; respectively) and living in a Serra city neighborhood decrease the risk of being in the 6-9 isolates/RFLP cluster (adjusted $\mathrm{OR}=0.29,95 \% \mathrm{Cl}, 0.10-0.84$ ), than in the others groups. Individuals aged 21 to 30, 31 to 40 and $>50$ years were less likely of belonging the 2-5 isolates/RFLP cluster than unique patterns compared to individuals $<20$ years of age (adjusted $\mathrm{OR}=0.49,95 \% \mathrm{Cl} 0.28-0.85, \mathrm{OR}=0.4395 \% \mathrm{Cl} 0$. 24-0.77and $\mathrm{OR}=0.49,95 \% \mathrm{Cl} 0.26-0.91$ ), respectively. The extrapulmonary disease was less likely to occur in those infected with strains in the $2-5$ isolates/cluster group (adjustment $\mathrm{OR}=0.45,95 \% \mathrm{Cl} 0.24-0.85$ ) than unique patterns.

Conclusions: We found that a large proportion of new TB infections in Vitoria is caused by prevalent Mtb genotypes belonging to the LAM family and RD Rio genotypes. Such information demonstrates that some genotypes are more likely to cause recent transmission. Targeting interventions such as screening in specific areas and social risk groups, should be a priority for reducing transmission.
\end{abstract}

Keywords: Tuberculosis, Molecular epidemiology, Transmission, Risk factors, Cluster size

\footnotetext{
* Correspondence: ethel.maciel@gmail.com

${ }^{2}$ Laboratório de Epidemiologia da Universidade Federal do Espírito Santo, Av.

Marechal Campos, 1468- Maruípe-, Vitória, ES, Brazil

Full list of author information is available at the end of the article
} 


\section{Background}

Tuberculosis (TB) continues to be a challenge to control. Although widespread and common efforts have had an impact in achieving declining numbers in global incidence for the first time in history, TB still causes 10.4 million new cases and 1.4 million deaths per year in worldwide [1].

Brazil ranks sixteenth among the world's 22 countries with high TB burdens; here, in 2016, the TB incidence was 63,189 cases, and the incidence rate was 30.9 per 100,000 per year, with mortality rate of 2.2 per 100,000 according to World Health Organization estimates [1]. The state of Espírito Santo has one of the lowest incidence of tuberculosis (28.6 / 100,000 / year) in Brazil [2]. Vitoria is a large urban setting and capital of Espírito Santo state, and reports over 279 cases of TB each year, which is among the highest incidence in the country (40.2/100,000/year) [3].

Understanding how TB transmission occurs is a key component to strategically manage TB from a public health perspective. Mycobacterium tuberculosis complex (MTBC) genotyping methods have been widely used in in molecular epidemiological studies [4]. These methods help to detect its spread, understand the dynamics of the disease, and develop tuberculosis (TB) control strategies to minimize TB expansion locally and globally.

Studies have suggested that identical IS6110 RFLP patterns of $M$. tuberculosis (Mtb) isolates from epidemiologically linked patients reflect TB resulting from recent transmission [5, 6]. Subsequent cases in transmission chains result in "clusters" of patients who share Mtb strains of the same genotype [7]. If a large proportion of new $\mathrm{TB}$ cases in a given community are due to recent transmissions, this is a reflection of an inadequate TB control program.

Many studies have investigated risk factors for clustering, suggesting that patient-related risk factors are important for $\mathrm{TB}$ transmission $[5,6,8]$. There is substantial evidence, however, that bacterial factors also contribute to variability in cluster size and the extent of transmission of $\mathrm{TB}$ in a community [8]. Indeed molecular epidemiologic studies have suggested that some strains are more successfully transmitted than others [9-11].

We performed genotyping of a large collection of Mtb strains that had been collected over a 11-year period in the metropolitan area of Vitoria, Espirito Santo state, Brazil and evaluated the relation between genotypes and clustering and strain and epidemiologic, clinical, and demographic characteristics. Our hypothesis was that this approach might help better understand risk factors for recent $\mathrm{TB}$ transmission in this particular setting.

\section{Methods}

\section{Study population}

This cross-sectional study examined all TB patients newly diagnosed in the metropolitan area of Vitoria, Brazil between 2000 and 2010. The Metropolitan area comprises four municipalities (Vitória, Cariacica, Serra and Vila Velha) with about 1,200,000 inhabitants. The study sample included isolates from all patients with positive culture results. The isolates were classified according to cluster size of Mtb strains and their associations with molecular and epidemiologic features were assessed.

\section{Genotyping methods}

\section{IS6110 restriction fragment length polymorphism (RFLP)} analysis

Sputum cultures for TB diagnosis are done routinely by the reference Mycobacteriology Laboratory at Núcleo de Doenças Infecciosas at the Federal University of Espirito Santo (NDI - UFES). We analyzed all available stored Mtb isolates that were consecutively obtained at reference laboratory for genotype analysis.

We used the standard IS6110 RFLP protocol [12] to genotype the isolates. Briefly, the genomic mycobacterial DNA was extracted, digested, and separated by gel electrophoresis. The DNA fragments resolved in agarose gel were transferred to a Hybond N-Plus membrane (GE Healthcare Life Sciences) and were hybridized with a probe made from a PCR product of the $3^{\prime}$ part of the PvuII fragment of IS6110. The IS6110 containing fragments on the membrane were detected by chemiluminescence (ECL direct ${ }^{\mathrm{TM}}$ nucleic acid labeling and detection system, GE Healthcare Limited, UK) and exposure to an X-ray film (A Hyperfilm ${ }^{\mathrm{TM}}$ ECL, GE Healthcare Limited, UK). The Mtb 14,323 strain was used as a reference strain for comparison of the RFLP patterns.

The IS6110 RFLP band patterns were analyzed by the BioNumerics software version 6.5 (Applied Maths Belgium). A dendrogram was constructed to show the degree of similarity among the isolates by unweighted pair group method of arithmetic average (UPGMA) and the Dice index (1.0\% tolerance, $1.5 \%$ optimization).

Two or more isolates with identical RFLP patterns (fingerprint) were defined as belonging to a cluster while strains with RFLP patterns of at least $70 \%$ similarity were considered members of the same "family". As described in other studies, isolates belonging to a cluster were considered to result from recent infections while isolates whose RFLP patterns were distinctly different from any other pattern identified among the isolates studied were considered unique or non-cluster patterns and were considered to represent reactivation from an old infection. Clusters composed patterns with less than six bands were tested by spoligotyping as this 
increases cluster reliability [13-16]. We named the clusters in our study with an abbreviation of ES, for Espírito Santo State.

Spoligotyping Isolates were also submitted to spoligotyping by a commercial kit (Ocimum Biosolutions Inc., India) according to a standard protocol $[17,18]$, allowing the classification of strains into spoligotype-based families, based on the presence or absence of spacer regions. Results were recorded in a 43-digit binary format and compared with an updated SpolDB4 [18] database - SITVITWEB [19] of the Pasteur Institute of Guadeloupe (available at http//:www.pasteur-guadeloupe.fr:8081/SIT VITDemo/) that provides information on the Mtb spoligotypes worldwide. The orphan patterns were entered into SPOTCLUST [20] in order to define the probability of a strain to belong to a certain family.

Long sequence polymorphism (LSP) A multiplex PCR adapted from Gibson et al. [21] was performed to identify isolates of the $\mathrm{RD}^{\text {Rio }}$ genotype. The differentiation of $\mathrm{RD}^{\text {Rio }}$ from non- $\mathrm{RD}^{\mathrm{Rio}}$ was determined according to the PCR product band size; the presence of a band of 1175 bp indicated $\mathrm{RD}^{\text {Rio }}$ while a band of 530-bp identified non- $\mathrm{RD}^{\text {Rio }}$ strains.

\section{Epidemiological, clinical and molecular characteristics}

We obtained general epidemiologic characteristics including gender, age, race, schooling (years), and previous history of TB, from the Brazilian national surveillance system (SINAN) and also from laboratory records maintained at the NDI-UFES. SINAN is the Brazilian Information System for notifiable diseases and its data are publicly accessible via the website of the Data Processing Department of Brazilian Ministry of Health (DATASUS) [22].

The following socio-demographic variables were evaluated: age $(<20$ years, $21-30$ years, $31-40$ years, $41-50$ years and $>50$ years), gender (male, female), race (white, black and others), and schooling ( $<4$ years, $4-8$ years, $>8$ years). The covariates related to TB included were: clinical form (PTB - pulmonary, EPTB - extra pulmonary, pulmonary + extra pulmonary), X-ray suspicious for TB (no, yes) and result of initial sputum smear (positive and negative). The genotype variables were those based on spoligotyping (LAM, non-LAM) and $\mathrm{RD}^{\text {Rio }}$ status $\left(\mathrm{RD}^{\mathrm{Rio}}\right.$, non- $\left.\mathrm{RD}^{\mathrm{Rio}}\right)$.

\section{Statistical analysis}

In order to identify risk factors for clustering, we performed univariate analysis using t-test for continuous variables and chi-square or the Fisher's exact test for categorical variables. Factors that were significantly associated with clustering were analyzed by a multiple logistic regression test by a stepwise approach to identify factors that were independent predictors of clustering.
We analyzed the isolates' distribution according to cluster size and observed that the sample was not normally distributed. Thus, we defined the "cluster size" into four categories (clusters with $2-5,6-9$ or $\geq 10$ isolates/cluster and unique patterns). Descriptive analysis of molecular and epidemiologic data was performed, according to cluster size classification. Based on a theoretical model for the study of determining TB [23], we performed crude analyses and we built one hierarchical polytomous regression model to identify factors associated with cluster size. Despite the categories of cluster size to imply an order, they did not meet the assumptions of an ordered logistic regression. Thus, we chose polytomous regression, which allows us to model simultaneously these multiple categories without the order assumption.

In the model we included all isolates analyzed. Unique pattern was defined as the reference group and was compared with the three cluster size categories.

The hierarchical levels for both models were defined as follows: level 1: the molecular variables (spoligotype and $\mathrm{RD}^{\text {Rio }}$ Genotype); level 2: the variables of level 1 and demographic variables (municipality of residence); level 3: the variables of level 2 and socio-demographic variables (age, gender, skin color and schooling); and level 4: the variables of level 3 and clinical variables (X-ray suspicious for $\mathrm{TB}$, result of initial sputum smear, and $\mathrm{TB}$ clinical form). Therefore, the total effect of each variable is adjusted for the variables at the same level and the levels above. Descriptive data were shown as absolute and relative frequencies or mean value and standard deviation. Results from association analysis were presented as odds ratios (OR) with confidence intervals of $95 \%$ (95\% CI). All analyses were conducted with the Stata statistical package, version 13.0 (StataCorp LP, College Station, TX, USA).

\section{Results}

Between January 2000 and December 2010, 5470 TB patients were diagnosed in the metropolitan area of Vitoria. Among these, 1320 (24\%) had culture performed, and we obtained good quality RFLP patterns from $959(72.6 \%)$ of them.

The IS6110 RFLP analysis demonstrated that 461 (48\%) cases had an isolate that belonged to a cluster and 498 (52\%) had a unique pattern (Table 1). Cluster size ranged from two to 34 isolates and 108 (11.2\%) formed a cluster with 10 or more isolates, 87 (9.1\%) with 6-9 isolates and $266(27.7 \%)$ with $2-5$ isolates.

All clusters were grouped into 30 RFLP families and six of these comprised $24.1 \%$ of the clustered isolates, while $108(11.2 \%)$ belonged to the six largest clusters ( $\geq 10$ isolates).

The cluster with the highest number of isolates was ES14 containing 34 followed by ES1b, ES8, ES14o, 
Table 1 Distribution of characteristics of TB patients according to their M. tuberculosis isolates' IS6110 RFLP cluster status

\begin{tabular}{|c|c|c|c|c|c|c|c|c|}
\hline \multirow[t]{2}{*}{ Characteristics } & \multirow[t]{2}{*}{ Categories } & \multicolumn{2}{|c|}{ All patients } & \multicolumn{2}{|l|}{ Cluster } & \multicolumn{2}{|c|}{ Unique Patterns (PU) } & \multirow{2}{*}{$\begin{array}{l}\text { Odds Ratio } \\
(95 \% \text { CI) }\end{array}$} \\
\hline & & $N=959$ & (\%) & $\overline{N=461}$ & (\%) & $N=498$ & $(\%)$ & \\
\hline \multicolumn{9}{|l|}{ Demographic } \\
\hline \multirow[t]{5}{*}{ Age (years old) } & $<20$ years & 100 & $(11.0)$ & 62 & $(14.4)$ & 38 & $(8.0)$ & 1 \\
\hline & $21-30$ years & 247 & $(27.3)$ & 115 & $(26.7)$ & 132 & $(27.9)$ & $0.53(0.33-0.85)$ \\
\hline & $31-40$ years & 220 & $(24.4)$ & 100 & $(23.2)$ & 120 & $(25.4)$ & $0.51(0.31-0.82)$ \\
\hline & $41-50$ years & 194 & $(21.5)$ & 93 & $(21.6)$ & 101 & $(21.4)$ & $0.56(0.34-0.92)$ \\
\hline & $>50$ years & 143 & $(15.8)$ & 61 & $(14.1)$ & 82 & $(17.3)$ & $0.45(0.27-0.76)$ \\
\hline \multirow[t]{2}{*}{ Gender } & Female & 287 & $(30.0)$ & 134 & $(29.0)$ & 153 & $(31.0)$ & 1 \\
\hline & Male & 672 & $(70.0)$ & 327 & $(71.0)$ & 345 & $(69.0)$ & $1.08(0.82-1.42)$ \\
\hline \multirow[t]{3}{*}{ Race } & White & 190 & $(30.0)$ & 90 & $(29.0)$ & 100 & $(31.0)$ & 1 \\
\hline & Black & 119 & $(19.0)$ & 64 & $(20.0)$ & 55 & $(17.0)$ & $1.29(0.81-2.04)$ \\
\hline & Others $^{a}$ & 323 & $(51.0)$ & 158 & $(51.0)$ & 165 & $(52.0)$ & $1.06(0.74-1.52)$ \\
\hline \multirow[t]{3}{*}{ Schooling (years) } & $\leq 4$ years & 106 & $(21.0)$ & 50 & $(21.0)$ & 56 & $(22.0)$ & 1 \\
\hline & 5 to 8 years & 304 & $(61.0)$ & 150 & $(62.0)$ & 154 & $(59.0)$ & $1.09(0.70-1.69)$ \\
\hline & $\geq 8$ years & 90 & $(18.0)$ & 41 & $(17.0)$ & 49 & $(19.0)$ & $0.93(0.53-1.64)$ \\
\hline \multirow[t]{5}{*}{ Metropolitan Area } & Vitoria & 583 & $(61.0)$ & 290 & $(63.0)$ & 293 & $(59.0)$ & 1 \\
\hline & Vila Velha & 89 & $(9.0)$ & 40 & $(9.0)$ & 49 & $(10.0)$ & $0.82(0.52-1.29)$ \\
\hline & Cariacica & 120 & $(13.0)$ & 60 & $(13.0)$ & 60 & $(12.0)$ & $1.01(0.68-1.49)$ \\
\hline & Serra & 123 & $(13.0)$ & 53 & $(11.0)$ & 70 & $(14.0)$ & $0.76(0.51-1.13)$ \\
\hline & Outros & 44 & $(5.0)$ & 18 & $(4.0)$ & 26 & $(5.0)$ & $0.70(0.37-1.30)$ \\
\hline \multicolumn{9}{|l|}{ Clinical } \\
\hline \multirow[t]{2}{*}{ X-Ray } & No & 46 & $(6.0)$ & 26 & $(8.0)$ & 20 & $(5.0)$ & 1 \\
\hline & Yes & 667 & $(94.0)$ & 318 & $(92.0)$ & 349 & $(95.0)$ & $0.99(0.96-1.03)$ \\
\hline \multirow[t]{3}{*}{ TB Presentation } & PTB $^{\mathrm{b}}$ & 779 & $(81.0)$ & 390 & $(85.0)$ & 389 & $(78.0)$ & 1 \\
\hline & EPTB & 111 & $(12.0)$ & 40 & $(9.0)$ & 71 & $(14.0)$ & $0.56(0.37-0.84)$ \\
\hline & $\mathrm{PTB}+\mathrm{EPTB}$ & 69 & $(7.0)$ & 31 & $(7.0)$ & 38 & $(8.0)$ & $0.81(0.49-1.33)$ \\
\hline \multirow[t]{2}{*}{ AFB Smears Results } & Negative & 147 & $(15.0)$ & 65 & $(14.0)$ & 82 & $(17.0)$ & 1 \\
\hline & Positive $^{c}$ & 802 & $(85.0)$ & 389 & $(86.0)$ & 413 & $(83.0)$ & $1.18(0.83-1.69)$ \\
\hline \multicolumn{9}{|l|}{ Molecular } \\
\hline \multirow[t]{2}{*}{ Spoligotyping } & Non LAM & 412 & $(48.0)$ & 195 & $(50.0)$ & 217 & $(46.0)$ & 1 \\
\hline & LAM & 448 & $(52.0)$ & 193 & $(50.0)$ & 255 & $(54.0)$ & $0.84(0.64-1.10)$ \\
\hline \multirow[t]{2}{*}{ RD ${ }^{\text {Rio }}$ Genotype } & Non - RD Rio & 552 & $(60.0)$ & 253 & $(58.0)$ & 299 & $(62.0)$ & 1 \\
\hline & $\mathrm{RD}^{\text {Rio }}$ & 369 & $(40.0)$ & 185 & $(42.0)$ & 184 & $(38.0)$ & $1.07(0.99-1.15)$ \\
\hline
\end{tabular}

a - Based on the SINAN response field for skin color

b - PTB = pulmonary TB, EPTB = extrapulmonary TB

c- All smear positive results were stratified as +1 (10-99 AFB/100 fields), +2 (1-10 AFB/field) and + 3 (> AFB/field)

d - Crude odds ratio from univariate logistic regression. $\mathrm{Cl}$, confidence interval

ES19h and ES25, which included 20, 16, 15, 13 and 10 isolates, respectively (Fig. 1). The ES14 genotype has an eight band pattern and is a member of the largest family $(n=86)$, sharing this pattern with one to three additional bands. In addition, the ES14 cluster has been present throughout the 11-year period and in $2003,68 \%$ of all $\mathrm{TB}$ cases belonging to the largest clusters were caused by three clonal groups ES14, ES19h and ES25. However, in 2007, the clusters ES14o, ES1b and ES8 contributed to $88 \%$ of all TB cases belonging to larger clusters.

We found that all isolates of the ES14 cluster were of the $\mathrm{RD}^{\text {Rio }}$ genotype while the other large clusters ES1b (20 isolates) and ES8 (16 isolates) were exclusively non$\mathrm{RD}^{\text {Rio }}(\mathrm{WT})$.

Of the total isolates spoligotyped, 448 (52\%) were classified as LAM and 412 (48\%) as non-LAM. Spoligotyping 


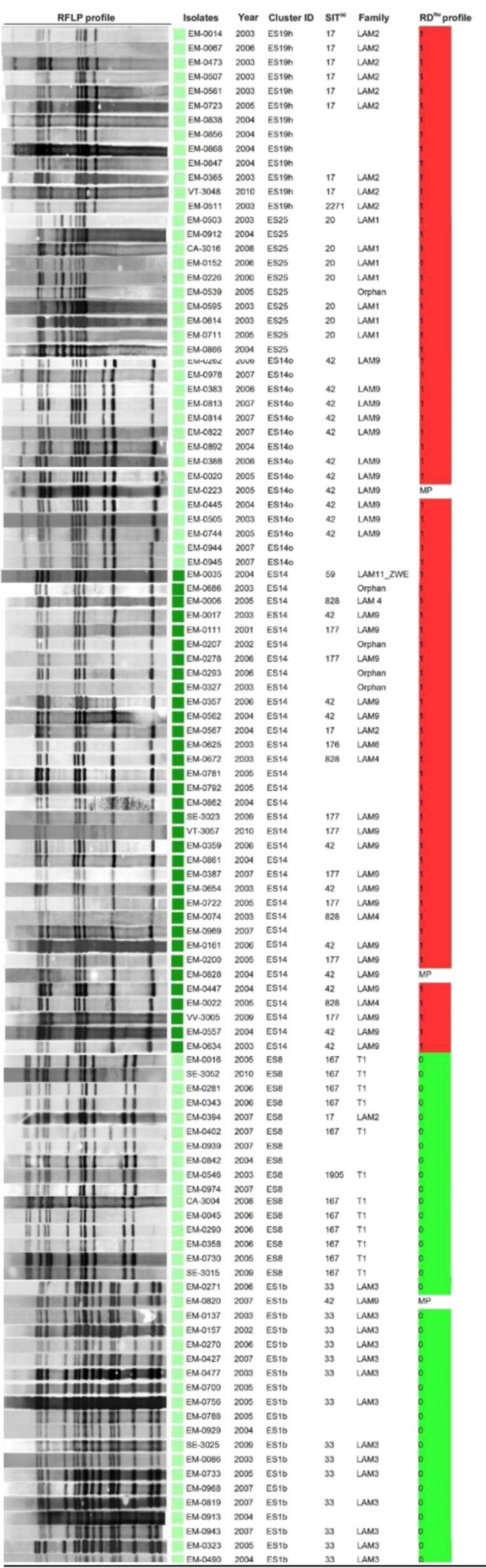

Fig. 1 Genotypic Profile of the six largest clusters found in the study. (a) - SIT (International Shared Type), (b) - RD Rio profile - (0): not $\mathrm{RD}^{\text {Rio }}$, (1): $R D^{\text {Rio }}$ and $(M P)$ : mixed population analysis for ES14 family showed one predominant sublineage (LAM9/SIT42; $n=42$ [42/86 = 49\%].

The results of univariate analysis of variables with genotype clustering status are presented in Table 1 . Patients with extrapulmonary TB (EPTB) group were less likely to be infected with a cluster strain than those with pulmonary $\mathrm{TB}$ (PTB) $(\mathrm{OR}=0.56$, 95\% CI $0.37-$ 0.84; OR $=0.98,95 \%$ CI $0.98-0.99$, respectively). Individuals aged 21 to 30 years, 31 to 40 years, $41-50$ years and $>50$ years were less likely to be infected with a cluster strain than compared to individuals $<20$ years of age $(\mathrm{OR}=0.53,95 \% \mathrm{CI} 0.33-0.85, \mathrm{OR}=0.5195 \% \mathrm{CI}$ $0.31-0.82, \mathrm{OR}=0.56,95 \% \mathrm{CI} 0.34-0.92$ and $\mathrm{OR}=0.45$, 95\% CI 0.27-0.76).

Table 2 summarizes the demographic, clinical characteristics and laboratory findings of $\mathrm{TB}$ patients with isolates belonging to a cluster $(2-5,6-9$ and $\geq 10)$ or a unique pattern genotype.

The hierarchical polytomous regression model (Table 3) showed that at the first level, those patients in the 6-9 and with $\geq 10$ isolates/cluster group were more likely to belong to the LAM lineage (adjusted OR $=1.17$, 95\% CI 1.08-1.26; adjusted OR $=1.25$, 95\% CI 1.14-1.37, respectively), using unique patterns as the reference.

On the other hand, subjects in the 2-5 isolates/cluster group were less likely to belong to the $\mathrm{RD}^{\text {Rio }}$ genotype (adjusted OR $=0.91,95 \%$ CI 0.80-1.03). At the second level, living in a specific neighborhood (Serra city) in the metropolitan area of Vitória decrease the risk of being in the 6-9 isolates/cluster group (adjusted OR $=0.29,95 \%$ CI, 0.10-0.84), using unique patterns as the reference group. In addition, at the third level individuals aged 21 to 30 years, 31 to 40 years and $>50$ years were less likely of belonging the 2-5 isolates/cluster group than unique patterns compared to individuals $<20$ years of age (adjusted $\mathrm{OR}=0.49,95 \%$ CI $0.28-0.85, \mathrm{OR}=0.4395 \% \mathrm{CI}$ $0.24-0.77$ and $\mathrm{OR}=0.49,95 \% \mathrm{CI} 0.26-0.91$ ) respectively.

Suspicion of TB on chest $\mathrm{x}$-ray was less likely observed for those infected with strains in the 2-5 isolates/cluster group (adjusted OR $=0.35,95 \%$ CI $0.15-0.79$ ) than unique patterns. Interestingly, the extrapulmonary disease was less likely to occur in those infected with strains in the 25 isolates/cluster group (adjustment $\mathrm{OR}=0.45,95 \% \mathrm{CI}$ 0.24-0.85) than unique patterns. Furthermore, TB patients whose isolates were included in the 6-9 isolates/cluster group (crude $\mathrm{OR}=2.68,95 \%$ IC 1.13-6.34) were more likely to be smear-positive compared to unique patterns as the reference. The difference was not statistically significant, however, after regression analysis.

\section{Discussion}

Since two decades ago, many studies on transmission of TB have been complemented by genotyping techniques. The IS6110 RFLP test has been used to distinguish 
Table 2 Distribution of characteristics of TB patients according to their M. tuberculosis isolates' IS6110 RFLP cluster size

\begin{tabular}{|c|c|c|c|c|c|c|c|c|c|c|c|}
\hline \multirow{3}{*}{ Characteristics } & \multirow{3}{*}{ Categories } & & & & & \multicolumn{6}{|c|}{ cluster size } \\
\hline & & \multicolumn{2}{|c|}{ All patients } & \multicolumn{2}{|l|}{$P U^{a}$} & \multicolumn{2}{|l|}{$2-5$} & \multicolumn{2}{|l|}{$6-9$} & \multicolumn{2}{|l|}{$\geq 10$} \\
\hline & & $N=959$ & (\%) & $N=498$ & (\%) & $N=266$ & (\%) & $N=87$ & (\%) & $N=108$ & $(\%)$ \\
\hline \multicolumn{12}{|l|}{ Demographic } \\
\hline \multirow[t]{5}{*}{ Age (years old) } & $<20$ years & 100 & (11.0) & 38 & (8.0) & 36 & $(14.6)$ & 17 & (19.8) & 9 & $(9.1)$ \\
\hline & $21-30$ years & 247 & (27.3) & 132 & $(27.9)$ & 60 & $(24.4)$ & 25 & $(29.1)$ & 30 & (30.3) \\
\hline & $31-40$ years & 220 & (24.4) & 120 & (25.4) & 50 & $(20.3)$ & 23 & $(26.7)$ & 27 & (27.3) \\
\hline & $41-50$ years & 194 & (21.4) & 101 & (21.4) & 60 & $(24.4)$ & 14 & $(16.3)$ & 19 & $(19.2)$ \\
\hline & $>50$ years & 143 & (15.8) & 82 & (17.4) & 40 & $(16.3)$ & 7 & $(8.2)$ & 14 & $(14.4)$ \\
\hline \multirow[t]{2}{*}{ Gender } & Female & 287 & (30.0) & 153 & (31.0) & 81 & $(30.0)$ & 24 & $(28.0)$ & 29 & $(27.0)$ \\
\hline & Male & 672 & $(70.0)$ & 345 & $(69.0)$ & 185 & $(70.0)$ & 63 & $(72.0)$ & 79 & (73.0) \\
\hline \multirow[t]{3}{*}{ Race } & White & 190 & (30.0) & 100 & (31.0) & 56 & $(32.0)$ & 20 & $(31.0)$ & 14 & (19.0) \\
\hline & Black & 119 & (19.0) & 55 & $(17.0)$ & 30 & $(17.0)$ & 14 & $(22.0)$ & 20 & $(27.0)$ \\
\hline & Others $^{b}$ & 323 & $(51.0)$ & 165 & (52.0) & 89 & $(51.0)$ & 30 & $(47.0)$ & 39 & $(53.0)$ \\
\hline \multirow[t]{3}{*}{ School Level (years) } & $\leq 4$ years & 106 & (21.0) & 56 & (22.0) & 32 & $(23.0)$ & 10 & $(20.0)$ & 8 & $(16.0)$ \\
\hline & $5-8$ years & 304 & (61.0) & 154 & $(59.0)$ & 86 & $(61.0)$ & 30 & $(61.0)$ & 34 & $(67.0)$ \\
\hline & $\geq 8$ years & 90 & (18.0) & 49 & (19.0) & 23 & $(16.0)$ & 9 & $(18.0)$ & 9 & $(18.0)$ \\
\hline \multirow[t]{5}{*}{ Metropolitan Area } & Vitória & 583 & (61.0) & 293 & $(59.0)$ & 162 & $(61.0)$ & 63 & $(72.0)$ & 65 & $(60.0)$ \\
\hline & Vila Velha & 89 & (9.0) & 49 & $(10.0)$ & 25 & $(9.0)$ & 7 & $(8.0)$ & 8 & $(7.0)$ \\
\hline & Cariacica & 120 & (13.0) & 60 & (12.0) & 34 & $(13.0)$ & 9 & $(10.0)$ & 17 & $(16.0)$ \\
\hline & Serra & 123 & (13.0) & 70 & (14.0) & 36 & $(14.0)$ & 4 & $(5.0)$ & 13 & $(12.0)$ \\
\hline & Others & 44 & $(5.0)$ & 26 & $(5.0)$ & 9 & (3.0) & 4 & $(5.0)$ & 5 & $(5.0)$ \\
\hline \multicolumn{12}{|l|}{ Clinical } \\
\hline \multirow[t]{2}{*}{ X Ray } & No & 46 & $(6.0)$ & 20 & $(5.0)$ & 17 & $(9.0)$ & 3 & $(4.0)$ & 6 & $(7.0)$ \\
\hline & Yes & 667 & (94.0) & 349 & (95.0) & 178 & $(91.0)$ & 65 & $(96.0)$ & 75 & $(93.0)$ \\
\hline \multirow[t]{3}{*}{ TB Presentation } & PTB $^{\mathrm{C}}$ & 779 & $(81.0)$ & 389 & (78.0) & 217 & $(82.0)$ & 79 & $(91.0)$ & 94 & $(87.0)$ \\
\hline & EPTB & 111 & (12.0) & 71 & (14.0) & 26 & $(10.0)$ & 6 & $(7.0)$ & 8 & $(7.0)$ \\
\hline & $\mathrm{PTB}+\mathrm{EPTB}$ & 69 & $(7.0)$ & 38 & (8.0) & 23 & $(9.0)$ & 2 & $(2.0)$ & 6 & $(6.0)$ \\
\hline \multirow[t]{2}{*}{ AFB Smears Results } & Negative & 147 & $(15.0)$ & 82 & $(17.0)$ & 48 & $(18.0)$ & 6 & $(7.0)$ & 11 & $(10.0)$ \\
\hline & Positive $^{d}$ & 802 & (85.0) & 413 & (83.0) & 214 & $(82.0)$ & 81 & $(93.0)$ & 94 & $(90.0)$ \\
\hline \multicolumn{12}{|l|}{ Molecular } \\
\hline \multirow[t]{2}{*}{ Spoligotyping } & Non LAM & 412 & $(48.0)$ & 217 & $(46.0)$ & 144 & $(61.0)$ & 33 & $(47.0)$ & 18 & $(22.0)$ \\
\hline & LAM & 448 & (52.0) & 255 & (54.0) & 91 & $(39.0)$ & 37 & $(53.0)$ & 65 & (78.0) \\
\hline \multirow[t]{2}{*}{ RD Rio Genotype } & Non RD ${ }^{\text {Rio }}$ & 552 & (60.0) & 299 & (62.0) & 182 & $(70.0)$ & 36 & $(50.0)$ & 35 & (33.0) \\
\hline & $\mathrm{RD}^{\text {Rio }}$ & 369 & (40.0) & 184 & (38.0) & 78 & $(30.0)$ & 36 & $(50.0)$ & 71 & (77.0) \\
\hline
\end{tabular}

a - When the unique patterns (PU) were the reference group and were compared with all others genotyped TB cases by

cluster size in number of patients per cluster

b - Based on the SINAN response field for skin colour

c_ PTB = pulmonary TB, EPTB = extrapulmonary TB

d. All smear positive results were stratified as +1 (10-99 AFB/100 fields), +2 (1-10 AFB/field) and + 3 (> AFB/field)

patients with TB due to recent transmission from reactivation disease [24]. In the present study, we evaluated transmission dynamics of TB in Vitoria, Brazil, during a 11-year period, comparing demographic, clinical and epidemiologic characteristics with Mtb genotypes and genotype clustering. We observed that a large proportion of recently transmitted TB was due to a limited set of Mtb genotypes and that certain cluster sizes were associated with patient demographic, clinical, or epidemiological characteristics.

A limitation of our study is that our patient data were derived from the SINAN secondary database with limited data. Data such as smoking, HIV status, drug abuse and drug susceptibilities testing, at the time of the study, 
Table 3 Crude and adjusted odds ratio by hierarchical polytomous regression analysis of the association of characteristics of TB patients according to their M. tuberculosis isolates' IS6110 RFLP cluster patterns (unique patterns for reference)

\begin{tabular}{|c|c|c|c|c|c|c|}
\hline & \multicolumn{3}{|l|}{ Crude OR } & \multicolumn{3}{|l|}{ Adjustment OR } \\
\hline & \multicolumn{3}{|l|}{ Cluster Size } & \multicolumn{3}{|l|}{ Cluster Size } \\
\hline & $2-5$ & $6-9$ & $\geq 10$ & $2-5$ & $6-9$ & $\geq 10$ \\
\hline & $\mathrm{OR}(\mathrm{Cl})$ & $\mathrm{OR}(\mathrm{Cl})$ & $\mathrm{OR}(\mathrm{Cl})$ & $\mathrm{OR}(\mathrm{Cl})$ & $\mathrm{OR}(\mathrm{Cl})$ & $\mathrm{OR}(\mathrm{Cl})$ \\
\hline \multicolumn{7}{|l|}{ Level 1} \\
\hline \multicolumn{7}{|l|}{ Spoligotyping } \\
\hline Non LAM & Ref & Ref & Ref & Ref & Ref & Ref \\
\hline LAM & $1.08(1.01-1.15)$ & $1.18(1.09-1.27)$ & $1.22(1.14-1.31)$ & $1.08(1.01-1.15)$ & $1.17(1.08-1.26)$ & $1.22(1.14-1.31)$ \\
\hline \multicolumn{7}{|l|}{ RD Rio Genotype } \\
\hline Non-RD Rio & Ref & Ref & Ref & Ref & Ref & Ref \\
\hline $\mathrm{RD}^{\mathrm{Rio}}$ & $0.91(0.81-1.03)$ & $1.26(1.15-1.38)$ & $1.06(0.94-1.19)$ & $0.91(0.80-1.03)$ & $1.25(1.14-1.37)$ & $1.05(0.93-1.18)$ \\
\hline \multicolumn{7}{|l|}{ Level 2} \\
\hline \multicolumn{7}{|l|}{ Metropolitan Area } \\
\hline Vitoria & Ref & Ref & Ref & Ref & Ref & Ref \\
\hline Vila Velha & $0.92(0.55-1.55)$ & $0.66(0.28-1.53)$ & $0.73(0.33-1.62)$ & $1.00(0.59-1.69)$ & $0.74(0.30-1.77)$ & $1.04(0.46-2.36)$ \\
\hline Cariacica & $1.02(0.64-1.62)$ & $0.70(0.32-1.47)$ & $1.27(0.69-2.33)$ & $1.12(0.70-1.80)$ & $0.71(0.32-1.58)$ & $1.81(0.96-3.40)$ \\
\hline Serra & $0.93(0.59-1.45)$ & $0.26(0.09-0.75)$ & $0.83(0.43-1.60)$ & $0.99(0.63-1.55)$ & $0.29(0.10-0.84)$ & $1.08(0.55-2.11)$ \\
\hline Outros & $0.62(0.28-1.36)$ & $0.71(0.24-2.12)$ & $0.86(0.32-2.34)$ & $0.66(0.30-1.45)$ & $0.74(0.24-2.28)$ & $1.04(0.37-2.89)$ \\
\hline \multicolumn{7}{|l|}{ Level 3} \\
\hline \multicolumn{7}{|l|}{ Gender } \\
\hline Female & Ref & Ref & Ref & Ref & Ref & Ref \\
\hline Male & $1.01(0.73-1.40)$ & $1.16(0.70-1.93)$ & $1.20(0.75-1.92)$ & $0.98(0.70-1.39)$ & $1.19(0.69-2.05)$ & $1.20(0.72-2.00)$ \\
\hline \multicolumn{7}{|l|}{ Age (years old) } \\
\hline$<20$ years & Ref & Ref & Ref & Ref & Ref & Ref \\
\hline $21-30$ years & $0.48(0.28-0.83)$ & $0.42(0.21-0.86)$ & $0.96(0.42-2.19)$ & $0.49(0.28-0.85)$ & $0.45(0.21-0.97)$ & $1.13(0.47-2.69)$ \\
\hline $31-40$ years & $0.44(0.25-0.77)$ & $0.43(0.21-0.88)$ & $0.95(0.41-2.19)$ & $0.43(0.24-0.77)$ & $0.41(0.19-0.90)$ & $1.01(0.42-2.43)$ \\
\hline $41-50$ years & $0.62(0.36-1.09)$ & $0.31(0.14-0.69)$ & $0.79(0.33-1.90)$ & $0.61(0.34-1.08)$ & $0.30(0.12-0.70)$ & $0.93(0.37-2.34)$ \\
\hline$>50$ years & $0.51(0.28-0.93)$ & $0.19(0.07-0.50)$ & $0.72(0.28-1.81)$ & $0.49(0.26-0.91)$ & $0.18(0.06-0.49)$ & $0.89(0.33-2.37)$ \\
\hline \multicolumn{7}{|l|}{ Race } \\
\hline White & Ref & Ref & Ref & Ref & Ref & Ref \\
\hline Black & $0.97(0.56-1.69)$ & $1.27(0.59-2.71)$ & $2.59(1.21-5.54)$ & $0.82(0.46-1.46)$ & $0.79(0.351 .81)$ & $2.02(0.90-4.51)$ \\
\hline Others & $0.96(0.63-1.46)$ & $0.90(0.49-1.68)$ & $1.68(0.87-3.26)$ & $0.89(0.58-1.38)$ & $0.76(0.39-1.49)$ & $1.53(0.77-3.05)$ \\
\hline \multicolumn{7}{|l|}{ School level, years } \\
\hline$\leq 4$ years & Ref & Ref & Ref & Ref & Ref & Ref \\
\hline 4 to 8 years & $0.97(0.58-1.62)$ & $1.09(0.50-2.37)$ & $1.54(0.67-3.53)$ & $0.89(0.52-1.51)$ & $1.01(0.42-2.41)$ & $1.49(0.62-3.57)$ \\
\hline$\geq 8$ years & $0.82(0.42-1.58)$ & $1.02(0.38-2.73)$ & $1.28(0.46-3.58)$ & $0.63(0.31-1.27)$ & $0.70(0.24-2.12)$ & $1.23(0.41-3.70)$ \\
\hline \multicolumn{7}{|l|}{ Level 4} \\
\hline \multicolumn{7}{|l|}{ X-ray } \\
\hline Negative & Ref & Ref & Ref & Ref & Ref & Ref \\
\hline Suspicious of TB & $0.60(0.30-1.17)$ & $1.24(0.35-4.29)$ & $0.71(0.27-1.84)$ & $0.34(0.14-0.79)$ & $0.35(0.08-1.56)$ & $0.27(0.08-0.92)$ \\
\hline \multicolumn{7}{|c|}{ Sputum smear results } \\
\hline Negative & Ref & Ref & Ref & Ref & Ref & Ref \\
\hline Positive & $0.88(0.60-1.31)$ & $2.68(1.13-6.34)$ & $1.69(0.87-3.30)$ & $0.69(0.41-1.17)$ & $2.05(0.68-6.16)$ & $1.24(0.53-2.90)$ \\
\hline
\end{tabular}

TB presentation 
Table 3 Crude and adjusted odds ratio by hierarchical polytomous regression analysis of the association of characteristics of TB patients according to their M. tuberculosis isolates' IS6110 RFLP cluster patterns (unique patterns for reference) (Continued)

\begin{tabular}{|c|c|c|c|c|c|c|}
\hline & \multicolumn{3}{|l|}{ Crude OR } & \multicolumn{3}{|l|}{ Adjustment OR } \\
\hline & \multicolumn{3}{|l|}{ Cluster Size } & \multicolumn{3}{|l|}{ Cluster Size } \\
\hline & $2-5$ & $6-9$ & $\geq 10$ & $2-5$ & $6-9$ & $\geq 10$ \\
\hline & $\mathrm{OR}(\mathrm{Cl})$ & $\mathrm{OR}(\mathrm{Cl})$ & $\mathrm{OR}(\mathrm{Cl})$ & $\mathrm{OR}(\mathrm{Cl})$ & $\mathrm{OR}(\mathrm{Cl})$ & $\mathrm{OR}(\mathrm{Cl})$ \\
\hline PTB & Ref & Ref & Ref & Ref & Ref & Ref \\
\hline EPTB & $0.65(0.40-1.05)$ & $0.41(0.17-0.99)$ & $0.46(0.21-1.00)$ & $0.44(0.23-0.83)$ & $0.41(0.13-1.21)$ & $0.37(0.13-1.03)$ \\
\hline $\mathrm{PTB}+\mathrm{EPTB}$ & $1.08(0.63-1.86)$ & $0.26(0.06-1.09)$ & $0.65(0.27-1.60)$ & $0.94(0.49-1.78)$ & $0.29(0.06-1.34)$ & $0.68(0.23-1.98)$ \\
\hline
\end{tabular}

OR odds ratio, $C l$ confidence interval, $S D$ standard deviation, $T B$ tuberculosis, $P T B$ pulmonary TB, EPTB extrapulmonary TB. *Hierarchical levels: Level $1=$

Spoligotyping $+\mathrm{RD}^{\text {Rio }}$ genotype; Level $2=$ Spoligotyping $+\mathrm{RD}^{\text {Rio }}$ genotype + Metropolitan Area; Level $3=$ Spoligotyping $+\mathrm{RD}$ Rio genotype + Metropolitan Area + Gender +Age + Race + Schooling; Level $4=$ Spoligotyping + RD Rio genotype + Metropolitan Area + Gender +Age + Race + Schooling + X-Ray + Sputum smear results + TB presentation

are not regularly reported by SINAN. On the other hand, the same database was the basis for studies on disease surveillance as described in earlier studies [11, 22, $25,26]$. The strength of the study is the large sample size, offering a statistical power that is higher than in most other studies. In addition, the long study period increases the chance of finding epidemiological links through genotyping of the Mtb strains, while that a small sample size and a poorly defined area can underestimate clustering proportions [27].

We provide evidence that six Mtb (cluster) strains have consistently contributed to the high burden of recent-transmission TB from 2000 to 2011 in the Metropolitan area of Vitória-ES. They accounted for $12 \%$ of all culture-confirmed TB cases in this area during this period. This observation was possible probably because of the high case coverage and length of the study period, increasing the chance of genotype clusters, as reported by van Soolingen et al. [28]. This finding is in concordance with a failing TB control program that fails to stop disease transmission in this area, and better approach should be implemented. This may be related to a variety of factors including delay in diagnosis and poor contact investigation strategies. Maciel and colleagues recently suggested a possibility for implementing a new casefinding strategy based on screening populations in neighborhoods with high-density recent-transmission TB and social network analyses [29].

In the present study, we found that isolates of the ES14 family accounted for the largest proportion of recently-transmitted TB cases, which suggests that these strains are either more transmissible or more likely to cause disease after infection. Strains of this family were LAM (mostly LAM9) family and $\mathrm{RD}^{\text {Rio }}$ genotype. Isolates with the particular eight band pattern that is the basis of this family have been reported as predominant also in studies conducted in Rio de Janeiro, São Paulo and Rio Grande do Sul in Brazil, and is frequently encountered in a database of isolates originating from other countries such as the Caribbean, Europe, Africa and other countries in South America [21, 30-32]. These findings suggest that the incidence of $\mathrm{TB}$ in this region may be strongly influenced by a relatively small subset of actively circulating strains. It is known that in areas with a higher incidence of TB, RFLP patterns are often less variable than in low-incidence areas [33]. Recently Ribeiro et al. (2015) showed that new TB cases do not just cluster in space, but that certain $M$. tuberculosis lineages tend to cluster even after controlling for known individual and socioeconomic factors that can influence transmission [29].

In our study, the clinical manifestation of $\mathrm{TB}-$ pulmonary or extrapulmonary $\mathrm{TB}$ - was associated with any particular cluster size (2-5 isolates/cluster). Although Gomes and colleagues showed no association between the clinical manifestation of TB and clustering rates, interestingly when stratified by cluster size a larger proportion of EPTB cases were in particular cluster size [25]. Our earlier studies also demonstrated that $\mathrm{RD}^{\text {Rio }}$ strains are less likely to cause extrapulmonary disease than non- $\mathrm{RD}^{\text {Rio }}$ strains [11] and the high prevalence of $\mathrm{RD}^{\text {Rio }}$ strains in the present study could be associated with levels of clustering of EPTB cases but many other factors influence clinical manifestations of TB, including the duration of illness before diagnosis as well as underlying host factors.

Our findings are consistent with several studies which have demonstrated the predominance of isolates of the LAM family and of the $\mathrm{RD}^{\text {Rio }}$ lineage in $\mathrm{TB}$ cases in Brazil [9, 11, 34-36]. Lazzarini and colleagues showed that the LAM1 and LAM2 sublineages exclusively belonged to the $\mathrm{RD}^{\text {Rio }}$ genotype, while the lineages LAM4, LAM5, LAM6 and LAM9 included both RD Rio and non $\mathrm{RD}^{\text {Rio }}$ genotypes, and LAM3 were all non$\mathrm{RD}^{\text {Rio }}$ [9], although some exceptions on this rule were presented recently [32]. Indeed, previous studies showed that $\mathrm{RD}^{\mathrm{Rio}}$ genotype is significantly associated with cluster groups (an indication of recent transmission) than non- $\mathrm{RD}^{\text {Rio }}$ strains, both in Brazilians and in nonBrazilian populations [36]. These data corroborate with 
findings in our study that showed that isolates from $\mathrm{RD}^{\text {Rio }}$ genotype belong to 6-9 isolates/cluster group. Although the proportion of cases with more 6-9 isolates/cluster patterns among $\mathrm{RD}^{\text {Rio }}$ strains was significantly greater than that non $\mathrm{RD}^{\text {Rio }}$ strains, it is not clear if this difference could be attributed to enhanced virulence and transmissibility of the $\mathrm{RD}^{\text {Rio }}$ strains. Previous studies suggested that these strains were recently introduced in some regions of Brazil and evolved after its introduction, or that the $\mathrm{RD}^{\text {Rio }}$ strains are more biologically "fit" [11, 36].

Glynn and colleagues suggested that these cluster strains are particularly transmissible or particularly more likely to cause disease after infection [36]. Other possibilities for their predominance are that they have been present in a geographic setting longer than others and that they had more time to become widespread, or that we are seeing a founder effect in some populations with subsequent spread following human migration patterns [33].

\section{Conclusions}

Our findings suggest that strains belonging to the LAM family and $\mathrm{RD}^{\text {Rio }}$ genotype showed are more likely to be largest clusters (6-9 and $\geq 10$ isolates/ cluster). We confirmed that the ES14 family is the most prevalent genotype of Mtb in Vitória - ES, Brazil, this suggesting, a large proportion of TB cases in one city can be caused by a few set of lineages circulating in the city. This provides an opportunity to characterize factors that affect transmission instead of host factors. Therefore, once an M. tuberculosis lineage enters in a community, that particular strain and its related family strains are more likely to propagate than outside strains.

\footnotetext{
Abbreviations

AFB: Acid-Fast Bacilli; Cl: Confidence interval; DATASUS: Department of Brazilian Ministry of Health; DNA: Deoxyribonucleic Acid; EPTB: Extrapulmonary TB; ES: abbreviation for clusters in Espirito Santo; IS6110: Insertion sequence 6110; LAM: Latin American Mediterranean; LSP: Long Sequence Polymorphism; Mtb: Mycobacterium tuberculosis; MTBC: Mycobacterium tuberculosis complex; NDI/UFES: Núcleo de Doenças Infecciosas at the Federal University of Espirito Santo; OR: Odds ratio; PCR: Polymerase Chain Reaction; PTB: Pulmonary TB; Pvu II: restriction endonuclease type II; RD: Regions Differences; RFLP: Restriction fragment length polymorphism; SINAN: Brazilian national surveillance system; TB: Tuberculosis; UPGMA: Unweighted pair group method of arithmetic average
}

\section{Acknowledgements}

Not applicable

\section{Funding}

This study was supported by Conselho Nacional de Desenvolvimento Científico and Tecnológico- CNPq by Edital MCT/CNPq/MS-SCTIE-DECIT N ${ }^{\circ}$ 25/2006 - Doenças negligenciadas, National Institutes of Health on the ICOHRTA grant \# 5 U2R TW006883-02 and CAPES (Coordenação de Aperfeiçoamento de Pessoal de Nível Superior).

\section{Availability of data and materials}

The data used for this study is not publicly available. For additional information about data and materials please contact the corresponding author for access to the data used in these analyses.

\section{Authors' contributions}

All authors made substantive intellectual contributions to the study. RLP, EM conceived and designed the study. RLP, SAV, FKR performed the experiments. EM, RLP, TNP, BRS, EZ analyzed the data and revised the manuscript. PNS, LWR, MP, SAV, JEG critically reviewed and revised the manuscript. RLP and EM was involved in the acquisition of the data, analysis and interpretation of data, and revision of the manuscript. All authors read and approved the final manuscript.

\section{Ethics approval and consent to participate}

Sputum culturing of individuals suspected to have TB is done routinely at clinics in the Vitória metropolitan area and the TB Reference Laboratory located at the Infectious Diseases Laboratory of the Federal University of Espirito Santo (UFES). The Mtb isolates are routinely stored by this laboratory for use in outbreak investigations and epidemiologic surveillance. This study was a retrospective analysis of data collected routinely during activities of the state TB control program. Patients were not contacted to request additional information. The study was reviewed and approved by the institutional review board of Universidade Federal do Espírito Santo (UFES; under number 121/06) who granted permission for use of the Mtb isolates and clinical data for the purposes of the study and waived the need for written informed consent from participants as the study involved no more than minimal risk and was done with existing microbiology specimens. Patients had an identification number for clinical purposes while cultures had a different accession number for laboratory purposes. To protect patient confidentiality, only one investigator (ELM) had access to both de-identification codes and was the person that linked of the clinical and culture databases for this study. After linkage, a new code number was created for each record for use in the study analysis.

\section{Consent for publication}

Not applicable

\section{Competing interests}

The author(s) declare that they have no competing interests.

\section{Publisher's Note}

Springer Nature remains neutral with regard to jurisdictional claims in published maps and institutional affiliations.

\section{Author details}

${ }^{1}$ Núcleo de Doenças Infecciosas, Universidade Federal do Espírito Santo, Vitória, Espirito Santo, Brazil. ${ }^{2}$ Laboratório de Epidemiologia da Universidade Federal do Espírito Santo, Av. Marechal Campos, 1468- Maruípe-, Vitória, ES, Brazil. ${ }^{3}$ Programa de Pós-Graduação em Epidemiologia, Universidade Federal de Pelotas, Pelotas, Brazil. ${ }^{4}$ Laboratório de Biologia Molecular Aplicada a Micobactérias, Instituto Oswaldo Cruz - FioCruz, Rio de Janeiro, Brazil.

${ }^{5}$ School of Medicine, Johns Hopkins University, Baltimore, MD, USA. ${ }^{6}$ Division of Infectious Disease and Vaccinology, School of Public Health, University of California, Berkeley, CA, USA.

Received: 28 August 2016 Accepted: 17 January 2018 Published online: 08 February 2018

References

1. World Health Organization (2016). Global tuberculosis report 2016. Available: http://www.who.int/tb/publications/global_report/en/index. html. Accessed 9 Jul 2017.

2. Ministério da Saúde. Boletim Epidemiológico Secretaria de Vigilância em Saúde - Perspectivas brasileiras para o fim da tuberculose como problema de saúde pública. 2016; 47 (13): 1-15.

3. Ministério da Saúde. Informações de saúde (TABNET) [Internet]. Brasília: Ministério da Saúde; 2016. Available: http://tabnet.datasus.gov.br/cgi/tabcgi. exe?sinannet/cnv/tuberces.def. Accessed 3 Jul 2017.

4. Perdigão J, Clemente S, Ramos J, Masakidi P, Machado D, Silva C, Couto I, Viveiros M, Taveira N, Portugal I. Genetic diversity, transmission dynamics 
and drug resistance of Mycobacterium tuberculosis in Angola. Sci Rep. 2017;23:7. 42814

5. Alland D, Kalkut GE, Moss AR, McAdam RA, Hahn JA, Bosworth W, Drucker E, Bloom BR. Transmission of tuberculosis in new York City. An analysis by DNA fingerprinting and conventional epidemiologic methods. N Engl J Med. 1994;330(24):1710-6.

6. Small PM, Hopewell PC, Singh SP, Paz a, Parsonnet J, Ruston DC, Schecter GF, Daley CL, Schoolnik GK. The epidemiology of tuberculosis in San Francisco. A population-based study using conventional and molecular methods. N Engl J Med. 1994;330(24):1703-9.

7. Nebenzahl-Guimaraes H, Borgdorff MW, Murray MB, van Soolingen D. A novel approach - the propensity to propagate (PTP) method for controlling for host factors in studying the transmission of mycobacterium tuberculosis. PLoS One. 2014;9(5):e97816. https://doi.org/10.1371/journal.pone.0097816.

8. LM V, van den Hof S, van Deutekom H, Hermans PW, Kremer K, Borgdorff MW, van Soolingen D. Mycobacterial factors relevant for transmission of tuberculosis. J Infect Dis. 2011;203(9):1249-55.

9. Lazzarini LCO, Huard RC, Boechat NL, Gomes HM, Oelemann MC, Kurepina N, Shashkina E, Mello FC, Gibson AL, Virginio MJ, Marsico AG, Butler WR, Kreiswirth BN, Suffys PN, Lapa E Silva JR, Ho JL. Discovery of a novel Mycobacterium tuberculosis lineage that is a major cause of tuberculosis in Rio de Janeiro, Brazil. J Clin Microbiol. 2007;45(12):3891-902

10. Teeter LD, Ha NP, Ma X, Wenger J, Cronin WA, Musser JM, Graviss EA. Evaluation of large genotypic mycobacterium tuberculosis clusters: contributions from remote and recent transmission. Tuberculosis. 2013;93(Suppl):S38-46.

11. Vinhas SA, Palaci M, Marques HS, Lobo de Aguiar PP, Ribeiro FK, Peres RL, Dietze R, Gomes HM, Suffys PN, Golub JE, Riley LW, Maciel EL. Mycobacterium tuberculosis DNA fingerprint clusters and its relationship with RD Rio genotype in Brazil. Tuberculosis. 2012;93(2):207-12.

12. Van Embden JD, Cave MD, Crawford JT, Dale JW, Eisenach KD, Gicquel B, Hermans P, Martin C, McAdam R, Shinnick TM, small PM. Strain identification of Mycobacterium tuberculosis by DNA fingerprinting: recommendations for a standardized methodology. J Clin Microbiol. 1993;31(2):406-9.

13. Varma-Basil M, Kumar S, Arora J, Angrup A, Zozio T, Banavaliker JN, Singh UB, Rastogi N, Bose M. Comparison of spoligotyping, mycobacterial interspersed repetitive units typing and IS6110-RFLP in a study of genotypic diversity of mycobacterium tuberculosis in Delhi, North India. Mem Inst Oswaldo Cruz. 2011;106(5):524-35.

14. Cowan LS, Diem L, Monson T, Wand P, Temporado D, Oemig TV, Crawford JT. Evaluation of a two-step approach for large-scale, prospective genotyping of mycobacterium tuberculosis isolates in the United States. J Clin Microbiol. 2005;43(2):688-95

15. Rhee JT, Tanaka MM, Behr MA, Agasino CB, Paz EA, Hopewell PC, small PM. Use of multiple markers in population based molecular epidemiologic studies of tuberculosis. Int J Tuberc Lung Dis. 2000;4(12):1111-9.

16. Van Soolingen D, de Haas PE, Hermans PW, Groenen PM, Van Embden JD. Comparison of various repetitive DNA elements as genetic markers for strain differentiation and epidemiology of Mycobacterium tuberculosis. J Clin Microbiol. 1993;31(8):1987-95.

17. Kamerbeek J, Schouls L, Kolk A, Van Agterveld M, Van Soolingen D, Kuijper S, Bunschoten A, Molhuizen H, Shaw R, Goyal M, van Embden J. Simultaneous Detection and strain differentiation of Mycobacterium tuberculosis for diagnosis and epidemiology. J Clin Microbiol. 1997;35(4):907-14.

18. Brudey K, Driscoll R, Rigouts L, Prodinger W, Gori A, Al-Hajoj SA, Allix C, Aristimuño L, Arora J, Baumanis V, Binder L, Cafrune P, Cataldi A, Cheong S, Diel R, Ellermeier C, Evans JT, Fauville-Dufaux M, Ferdinand S, Garcia de Viedma D, Garzelli C, Gazzola L, Gomes HM, Guttierez MC, Hawkey PM, van Helden PD, Kadival GV, Kreiswirth BN, Kremer K, Kubin M, Kulkarni SP, Liens B, Lillebaek T, Ho ML, Martin C, Martin C, Mokrousov I, Narvskaïa O, Ngeow YF, Naumann L, Niemann S, Parwati I, Rahim Z, Rasolofo-Razanamparany V, Rasolonavalona T, Rossetti ML, Rüsch-Gerdes S, Sajduda A, Samper S, Shemyakin IG, Singh UB, Somoskovi A, Skuce RA, van Soolingen D, Streicher EM, Suffys PN, Tortoli E, Tracevska T, Vincent V, Victor TC, Warren RM, Yap SF, Zaman K, Portaels F, Rastogi N, Sola C. Mycobacterium tuberculosis complex genetic diversity: mining the fourth international spoligotyping database (SpolDB4) for classification, population genetics and epidemiology. BMC Microbiology. 2006;6:6-23.

19. Demay C, Liens B, Burguière T, Hill V, Couvin D, Millet J, Mokrousov I, Sola C, Zozio T, Rastogi N. SITVITWEB- a publicly available international multimarker database for studying mycobacterium tuberculosis genetic diversity and molecular epidemiology. Infect Genet Evol. 2012;12(4):755-66.
20. Vitol I, Driscoll J, Kreiswirth B, Kurepina N, Bennett KP. Identifying mycobacterium tuberculosis complex strain families using spoligotypes. Infect Genet Evol. 2006;6(6):491-504.

21. Gibson AL, Huard RC, Gey van Pittius NC, Lazzarini LC, Driscoll J, Kurepina N, Zozio T, Sola C, Spindola SM, Kritski AL, Fitzgerald D, Kremer K, Mardassi H, Chitale P, Brinkworth J, Garcia de Viedma D, Gicquel B, Pape JW, van Soolingen D, Kreiswirth BN, Warren RM, van Helden PD, Rastogi N, Suffys PN, Lapa e Silva J, Ho JL. Application of sensitive and specific molecular methods to uncover global dissemination of the major RDRio sublineage of the Latin American-Mediterranean Mycobacterium tuberculosis spoligotype family. J Clin Microbiol. 2008:46(4):1259-67.

22. Malhão TA, Oliveira GP, Codennoti SB, Moherdaui F. Evaluation of data completeness in the tuberculosis notification information system, Brazil, 2001-2006. Epidemiol Serv Saúde. 2010;3:245-56.

23. Maciel EL, Reis-Santos B. Determinants of tuberculosis in Brazil: from conceptual framework to practical application. Rev Panam Salud Publica. $2015 \mathrm{Jul} ; 38(1): 28-34$.

24. Coscolla M, Gagneux S. Does M. tuberculosis genomic diversity explain disease diversity? Drug Discov today dis Mech. Spring. 2010;7(1):e43-9.

25. Gomes T, Vinhas SA, Reis-Santos B, Palaci M, Peres RL, Aguiar PP, Ribeiro FK, Marques HS, Dettoni Vdo V, Johnson JL, Riley LW, Maciel EL. Extrapulmonary tuberculosis: mycobacterium tuberculosis strains and host risk factors in a large urban setting in Brazil. PLoS One. 2013;8(10):e74517.

26. Moreira CM, Maciel EL. Completeness Of tuberculosis control program records in the case registry database of the state of Espirito Santo, Brazil: analysis of the 2001-2005 period. J Bras Pneumol. 2008:34(4):225-9.

27. Houben RM, Glynn JR. A systematic review and meta-analysis of molecular epidemiological studies of tuberculosis: development of a new tool to aid interpretation. Tropical Med Int Health. 2009;14:892-909.

28. Van Soolingen D, Borgdorff MW, de Haas PE, Sebek MM, Veen J, Dessens M, Kremer K, Van Embden JD. Molecular epidemiology of tuberculosis in the Netherlands: a nationwide study from 1993 through 1997. J Infect Dis. 1999; 180(3):726-36

29. Ribeiro FK, Pan W, Bertolde A, Vinhas SA, Peres RL, Riley L, Palaci M, Maciel EL. Genotypic and Spatial Analysis of Mycobacterium tuberculosis Transmission in a High-Incidence Urban Setting. Clin Infect Dis. 2015; 5. pii: civ365.

30. Suffys PN, Ivens de Araujo ME, Rossetti ML, Zahab A, Barroso EW, Barreto AM, Campos E, van Soolingen D, Kremer K, Heersma H, Degrave WM. Usefulness of IS6110-restriction fragment length polymorphism typing of Brazilian strain of mycobacterium tuberculosis and comparison with an international fingerprint database. Res Microbiol. 2000;151(5):343-51.

31. Baptista IM, Oelemann MC, Opromolla DV, Suffys PN. Drug resistance and genotypes of strains of mycobacterium tuberculosis isolated from human immunodeficiency virus-infected and non-infected tuberculosis patients in Bauru, São Paulo, Brazil. Mem Inst Oswaldo Cruz. 2002;97(8):1147-52.

32. Vasconcellos SE, Acosta CC, Gomes LL, Conceição EC, Lima KV, de Araujo MI, Leite Mde L, Tannure F, Caldas PC, Gomes HM, Santos AR, Gomgnimbou MK, Sola C, Couvin D, Rastogi N, Boechat N, Suffys PN. Strain classification of Mycobacterium tuberculosis isolates in Brazil based on genotypes obtained by spoligotyping, mycobacterial interspersed repetitive unit typing and the presence of large sequence and single nucleotide polymorphism. PLoS One. 2014;9(10):e107747.

33. van Soolingen D, Hermans PW. Epidemiology of tuberculosis by DNA fingerprinting. Eur Respir J Suppl. 1995; (20): 649s-656s. Review.

34. Gomes HM, Elias AR, Oelemann MA, Pereira MA, Montes FF, Marsico AG, Kritski AL, Filho Ldos A, Caldas PC, Possuelo LG, Cafrune P, Rossetti ML, Lucena N, Saad MH, Cavalcanti HR, Leite CQ, de Brito RC, Lopes ML, Lima K, Souza M, Trindade Rde C, Zozio T, Sola C, Rastogi N, Suffys PN. Spoligotypes of Mycobacterium tuberculosis complex isolates from patients residents of 11 states of Brazil. Infect Genet Evol. 2012;12(4):649-56.

35. Weisenberg S A, Gibson A L, Huard R C, Kurepina N, Bang H, Lazzarini LC, Chiu Y, Li J, Ahuja S, Driscoll J, Kreiswirth BN, Ho JL. Distinct clinical and epidemiological features of tuberculosis in new York City caused by the RD (Rio) Mycobacterium tuberculosis sublineage. Infect Genet Evol. 2012; 12 (4): 664-670.

36. Lazzarini LCO, Spindola SM, Bang H, Gibson AL, Weisenberg S, da Silva Carvalho W, Augusto CJ, Huard RC, Kritski AL, Ho JL. RD ${ }^{\text {Rio }}$ mycobacterium tuberculosis infection is associated with a higher frequency of cavitary pulmonary disease. J Clin Microbiol. 2008;46(7):2175-83. 